\title{
Black holes as bubble nucleation sites
}

\author{
Ruth Gregory, ${ }^{a, b}$ lan G. Moss ${ }^{c}$ and Benjamin Withers ${ }^{a, d}$ \\ ${ }^{a}$ Department of Mathematical Sciences and Centre for Particle Theory, \\ South Road, Durham, DH1 3LE, U.K. \\ ${ }^{b}$ Perimeter Institute, 31 Caroline Street North, \\ Waterloo, ON, N2L 2Y5, Canada \\ ${ }^{c}$ School of Mathematics and Statistics, Newcastle University, \\ Newcastle Upon Tyne, NE1 7RU, U.K. \\ ${ }^{d}$ Mathematical Sciences and STAG research centre, University of Southampton, \\ Higheld, Southampton SO17 1BJ, U.K. \\ E-mail: r.a.w.gregory@durham.ac.uk, ian.moss@newcastle.ac.uk, \\ b.s.withers@soton.ac.uk
}

ABStract: We consider the effect of inhomogeneities on the rate of false vacuum decay. Modelling the inhomogeneity by a black hole, we construct explicit Euclidean instantons which describe the nucleation of a bubble of true vacuum centred on the inhomogeneity. We find that inhomogeneity significantly enhances the nucleation rate over that of the Coleman-de Luccia instanton - the black hole acts as a nucleation site for the bubble. The effect is larger than previously believed due to the contributions to the action from conical singularities. For a sufficiently low initial mass, the original black hole is replaced by flat space during this process, as viewed by a single causal patch observer. Increasing the initial mass, we find a critical value above which a black hole remnant survives the process. This resulting black hole can have a higher mass than the original black hole, but always has a lower entropy. We compare the process to bubble-to-bubble transitions, where there is a semi-classical Lorentzian description in the WKB approximation.

Keywords: Cosmology of Theories beyond the SM, Black Holes, Solitons Monopoles and Instantons

ARXIV EPRINT: 1401.0017 


\section{Contents}

1 Introduction 1

2 Lorentzian and Euclidean thin-wall space-times 4

2.1 Lorentzian bubbles 5

2.2 Euclidean bubbles 8

3 Computing the Euclidean action $\quad 9$

$\begin{array}{ll}3.1 \text { General results } & 10\end{array}$

4 Tunnelling from the false vacuum $\quad \mathbf{1 2}$

$\begin{array}{lll}4.1 & \text { Coleman de Luccia bubbles } & 13\end{array}$

4.2 The critical static bubble wall with $M_{-}=0 \quad 13$

4.3 General $M_{-}=0$ bubbles 14

4.4 The critical static bubble wall with $M_{-} \neq 0 \quad 14$

$\begin{array}{ll}4.5 \text { The dominant processes } & 15\end{array}$

5 The WKB approach 16

$\begin{array}{llr}6 & \text { Discussion } & 19\end{array}$

$\begin{array}{ll}\text { A Conical deficit regularisation } & 22\end{array}$

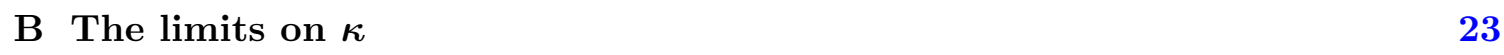

\section{Introduction}

One of the most exciting aspects of quantum field theory is the possibility that the universe can become trapped in a false vacuum state. For first order phase transitions, the decay rate of the false vacuum state is exponentially suppressed, [1,2], allowing for a long-lived metastable state with consequences for the very early universe, [3], or the possible fate of the late universe, [4].

Aside from cosmology, the decay rates for most commonly observed first order phase transitions are greatly enhanced by the presence of nucleation sites for the preferred low temperature phase, such as impurities or imperfections in the retaining walls. The goal of this paper is to explore the cosmological version of a nucleation site by considering false vacuum decay in the presence of inhomogeneities. Enhancing the transition rate could prevent the universe becoming trapped in a false vacuum state or in a worst case scenario could bring about the premature end of the universe. 
There have been very few previous investigations of the nucleation rates of true vacuum bubbles around black holes, the closest to our work being Hiscock, [5]. Berezin et al. also investigated false vacuum decay around black holes in de Sitter space, [6, 7]. The nucleation of a symmetric phase around an evaporating black hole was investigated in [8]. Some recent work has been done on false vacuum decay due to modifications of the scalar field potential by the black hole, [9].

A prototypical example of false vacuum decay, described by the Coleman-de Luccia (CDL) instanton, [10], takes place in the idealised setting of a maximally symmetric false vacuum universe - de Sitter space-time. We will relax the initial condition of a homogeneous universe, and show that introducing inhomogeneity enhances the rate of production of true vacuum bubbles centred on the inhomogeneity. In particular, we consider the natural generalisation of the CDL Euclidean instanton solution to include the simplest form of inhomogeneity: a black hole. We are thus led to study the formation of vacuum bubbles in the false vacuum background described by the Schwarzschild-de Sitter black hole (SdS) metric:

$$
d s^{2}=-f(r) d t^{2}+\frac{d r^{2}}{f(r)}+r^{2} d \Omega_{2}^{2}, \quad f(r) \equiv 1-\frac{2 G M}{r}-\frac{r^{2}}{\ell^{2}},
$$

where the de Sitter radius, $\ell$, is related to the energy density of the false vacuum, $\varepsilon$, by the relation $\ell^{2}=3 /(8 \pi G \varepsilon)$. As usual, the two positive roots of $f(r)$ correspond to the locations of the black hole horizon, $r_{h}$, and of the cosmological horizon, $r_{c}$. The horizons coincide when $G M / \ell=G M_{N} / \ell \equiv 1 / \sqrt{27}$, which corresponds to the Nariai solution, [11, 12].

We shall construct the analogue of the thin-wall Euclidean 'bounce' solution in the presence of finite mass, $M$. For convenience we work with the Euclidean section obtained by performing a Wick rotation, $t=-i \tau$, from the causal patch of SdS described by (1.1), where we can take the coordinate $\tau$ to have period $\beta$. For an arbitrary choice of $\beta$ there are conical singularities at $r_{h}$ and $r_{c}$, the fixed points under the action of rotation in Euclidean time. In the thin-wall limit, an oscillatory bubble wall trajectory, $(\tau(\lambda), r(\lambda))$, describes the locus where we will match an exterior SdS solution with $r>r(\lambda)$ onto an interior true vacuum region with $r<r(\lambda)$. A sketch of the wall trajectory is shown in figure 1 . Note that because the Euclidean bubble wall trajectories are oscillatory in $\tau$ with their own characteristic period, $\beta_{\text {wall }}$, neither conical singularity can be removed with a choice for the period $\beta$ as we must choose $\beta=\beta_{\text {wall }}$. The field equations break down at the conical singularities, but despite this, a careful treatment of the conical singularities can be made, and the appropriate contribution to the Euclidean action computed. Finite action solutions with conical singularities are known in the literature as singular instantons, [13, 14].

In the cases where $\partial_{\tau}$ is a Killing vector - for example in the absence of the bubble wall, or for a $\tau$-independent bubble wall — we show that the Euclidean action is always independent of the choice of $\beta$ once the conical singularities are taken into account. This removes a possible ambiguity in the tunnelling rates when using singular instantons. Our formula for the actions of symmetric singular instantons generalises the 'NUTs and bolts' formula of Gibbons and Hawking, [15]. For the non-static bubble, we find precise agreement for the value of the action with two cases where we are aware of an equivalent, manifestly regular construction: the CDL instanton with zero mass, and a second special case at finite 


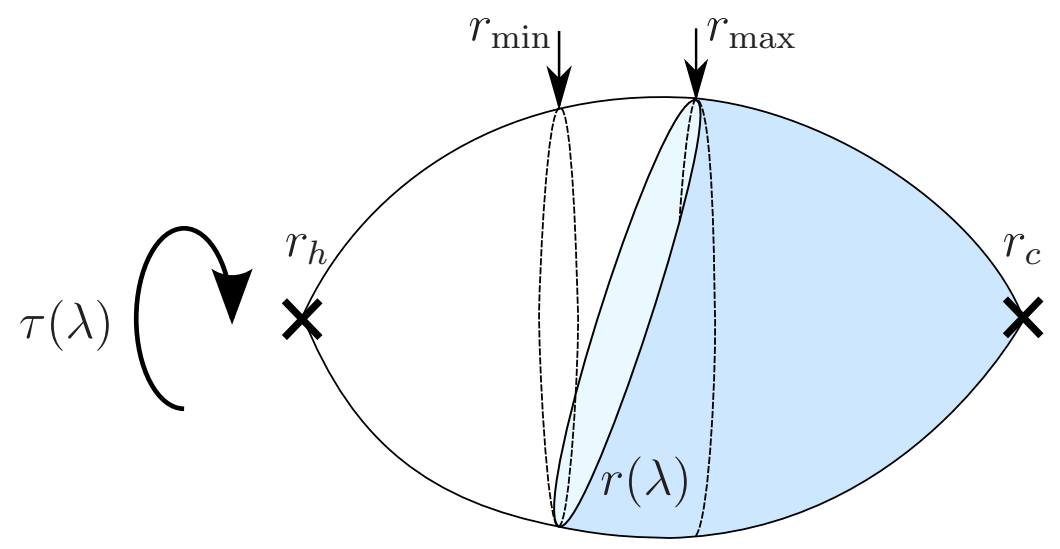

Figure 1. Cartoon of a thin-wall in the Euclidean continuation of SdS. For clarity, we display only the $\tau, r$ surface, and the $S^{2}$ is not shown. The shaded region indicates the false-vacuum exterior. The interior is not shown. The time circle has been identified with the period of the wall solution shown, leading to conical singularities at the fixed points indicated by the black crosses.

$M_{*}$ where there is a critical, $\tau$-independent bubble wall, corresponding to solutions found in $[16]$.

The main physical result of our analysis is the behaviour of the nucleation rate of true vacuum bubbles, $\Gamma(M)$, as a function of the initial black hole seed mass $M$. We find that there are two regimes, delineated by a critical mass,

$$
G M_{C}=\frac{32}{27} \frac{(8 \pi G \sigma \ell)^{3}}{\left(4+(8 \pi G \sigma \ell)^{2}\right)^{2}},
$$

where $\sigma$ is the tension of the thin-wall:

- For an initial seed black hole with $M<M_{C}$, the rate is an increasing function of the mass,

$$
\left.\frac{\partial \Gamma(M)}{\partial M}\right|_{\sigma}>0
$$

with the dominant instanton corresponding to the nucleation of flat space inside the bubble. The nucleation rate for a bubble of true vacuum which replaces a finite mass SdS black hole is actually higher than that of the CDL case. Although this conclusion agrees qualitatively with the previous work of Hiscock [5], there the contributions from conical singularities were not taken into account. The result here is a far larger increase in the bubble nucleation rate.

- For an initial seed black hole with mass $M>M_{C}$, the rate is a decreasing function of the mass and for sufficiently large black holes the rate eventually becomes subdominant to that of the CDL instanton. For this range we find that the dominant process corresponds to bubble nucleation with a vacuum black hole, which we shall here term 'the remnant'. The mass of this remnant black hole may be higher than $M$, but the horizon area is always smaller. 
- The critical value, $M=M_{C}$, marks the point at which the remnant black hole becomes vanishingly small when approached from above, giving way to flat space inside the bubble. Here, the transition rate is maximised, and is a decreasing function of the wall tension $\sigma$.

In contrast to the Euclidean instanton approach, in this paper we also consider the Lorentzian WKB method, [17-21], used to calculate the probability for transitions between bubble solutions: 'bubble-to-bubble' transitions. These methods have been used mostly to investigate the nucleation of false vacuum bubbles in the context of creating baby universes, [17]. The basic idea of the WKB method is to formulate an action which depends only on the bubble wall trajectory and then use the associated Schrödinger equation to calculate tunnelling probabilities. We present a new action for the bubble wall and show that in general, the bubble-to-bubble transition rate calculated via the WKB method is related to the spontaneous nucleation rate calculated in the singular instanton approach via a numerical factor depending only on the black hole entropy. We propose that this represents a type of crossing relation for the amplitude describing the bubble-to-bubble transition.

The paper is organised as follows. We begin in section 2 with a computation of the thin-wall trajectories in both the Lorentzian, (1.1), and Euclidean pictures. In section 3 we present a general derivation of the Euclidean action in the presence of conical singularities, which we then evaluate for the wall trajectories for generic values of the mass to compute nucleation rates in section 4 , where we also outline the dominant processes. In section 5 we elucidate the connection to the Lorentzian WKB approach for bubble-to-bubble transitions. We conclude in section 6 .

\section{Lorentzian and Euclidean thin-wall space-times}

We consider the following system of gravity and matter fields,

$$
S=\frac{1}{16 \pi G} \int_{\mathcal{M}} \mathcal{R}+\int_{\mathcal{M}} \mathcal{L}_{m}(g, \phi)
$$

where the manifold $\mathcal{M}$ has a metric $g$ of Lorentzian signature $(-,+,+,+)$ and Ricci scalar $\mathcal{R}$. For each vacuum present in this theory we can construct a one parameter family of SdS black hole solutions (1.1). The length scale $\ell$ is determined by the cosmological constant in the chosen vacuum, $\ell^{2}=3 / \Lambda$. In this Lorentzian system $(2.1)$ we are interested in constructing space-times which describe two vacua separated by a thin bubble wall, and as with CDL, we will use Israel's junction conditions, [22], to match a solution of the form (1.1) with mass $M_{-}$and cosmological constant $\Lambda_{-}$(the 'inside') across a thin bubble wall of tension $\sigma$ to a solution mass $M_{+}$and cosmological constant $\Lambda_{+}$(the 'outside'). These will in general be time-dependent bubble wall trajectories, many of which will correspond to a reflection or bounce.

We may also study Euclidean solutions obtained by performing the Wick rotation $t=-i \tau$ from the causal patch (1.1) to obtain a closed Euclidean manifold,

$$
d s^{2}=f(r) d \tau^{2}+\frac{d r^{2}}{f(r)}+r^{2} d \Omega_{2}^{2}, \quad f(r) \equiv 1-\frac{2 G M}{r}-\frac{r^{2}}{\ell^{2}},
$$


which solve the equations of motion coming from the corresponding Euclidean action given by $I=-i S$. Proceeding by analogy with the CDL instanton, we may also construct a family of Euclidean thin-wall solutions separating different vacuum solutions of the form (2.2). We shall later make the interpretation that the on-shell action for these solutions, $I$, determines the rate of bubble nucleation, just as in the CDL case. Indeed when $M_{-}=M_{+}=\Lambda_{-}=0$ we obtain the CDL result, however, unlike Coleman and de Luccia, we will work entirely in the Euclidean continuation of a single causal static patch of SdS i.e., (2.2).

\subsection{Lorentzian bubbles}

In the thin wall description of the bubble, we describe the trajectory of the wall by local coordinates on each side of the wall:

$$
X_{ \pm}^{a}=\left(t_{ \pm}(\lambda), r_{ \pm}(\lambda), \theta, \phi\right)
$$

where for convenience, we take $\lambda$ to be the proper time of an observer comoving with the wall,

$$
f_{ \pm}\left(r_{ \pm}\right) \dot{t}_{ \pm}^{2}-\frac{\dot{r}_{ \pm}^{2}}{f_{ \pm}\left(r_{ \pm}\right)}=1
$$

The intrinsic coordinates on the wall are $\xi^{A}=(\lambda, \theta, \phi)$, and the induced metric is

$$
d s^{2}=-d \lambda^{2}+r_{ \pm}^{2}(\lambda)\left[d \theta^{2}+\sin ^{2} \theta d \phi^{2}\right] .
$$

Clearly, if the wall is to make physical sense as a boundary between two regions, we require $r_{+}=r_{-} \equiv R(\lambda)$.

Next, we construct a normal one-form on each side of the wall

$$
n_{ \pm}=\left(-\dot{r}_{ \pm} d t_{ \pm}+\dot{t}_{ \pm} d r_{ \pm}\right)
$$

with the sign chosen so that it is always pointing towards increasing $r$ for $\dot{t}>0$. From these, we construct the extrinsic curvature of each side of the wall:

$$
K_{ \pm A B}=X_{ \pm, A}^{a} X_{ \pm, B}^{b} \nabla_{a} n_{ \pm b}
$$

Treating the wall's stress tensor $T_{a b}^{w}$ as a distributional source, we may construct the surface stress tensor,

$$
S_{a b} \equiv \int T_{a b}^{w} d l
$$

and the Israel junction conditions, [22], then relate the energy-momentum of the wall to the geometry of its embedding measured via a jump in the extrinsic curvature across the wall:

$$
\Delta K_{a b} \equiv K_{+a b}-K_{-a b}=-8 \pi G\left(S_{a b}-\frac{1}{2} h_{a b} S\right)
$$

For a wall of tension $\sigma$ we have $S_{a b}=-\sigma h_{a b}$ and this equation reduces to

$$
\frac{1}{R}\left(f_{+}(R) \dot{t}_{+}-f_{-}(R) \dot{t}_{-}\right)=-4 \pi G \sigma .
$$


Using (2.4), we can substitute for $\dot{t}_{ \pm}$on each side, and rearranging reveals a Friedman-like equation, [23], for the trajectory of the wall

$$
\left(\frac{\dot{R}}{R}\right)^{2}=\bar{\sigma}^{2}-\frac{\bar{f}}{R^{2}}+\frac{(\Delta f)^{2}}{16 R^{4} \bar{\sigma}^{2}} .
$$

where $\bar{\sigma} \equiv 2 \pi G \sigma, \bar{f} \equiv\left(f_{-}+f_{+}\right) / 2$, and $\Delta f \equiv f_{+}-f_{-}$. To completely determine the wall trajectory, we must determine the time coordinate evolution, obtained by combining (2.11) with (2.4), ensuring consistency with the Israel junction equation (2.10):

$$
f_{ \pm} \dot{t}_{ \pm}=\sqrt{f_{ \pm}+\dot{R}^{2}}=\mp \bar{\sigma} R-\frac{\Delta f}{4 \bar{\sigma} R}
$$

(2.11) and (2.12) now completely describe the bubble wall trajectory for general $M_{ \pm}, \Lambda_{ \pm}$.

In this paper we are primarily interested in the effects of mass on false vacuum decay, and for this purpose we consider the class of solutions where the interior solution is true vacuum, i.e. $\Lambda_{-}=0$, and the exterior is false vacuum, $\Lambda_{+}=3 / \ell^{2}$. This includes the CDL case, which simply has $M_{+}=M_{-}=0$. The radial equation (2.11) in this case becomes,

$$
\left(\frac{\dot{R}}{R}\right)^{2}+\frac{1}{R^{2}}=\left(\bar{\sigma}+\frac{1}{4 \bar{\sigma} \ell^{2}}+\frac{G \Delta M}{2 \bar{\sigma} R^{3}}\right)^{2}+\frac{2 G M_{-}}{R}
$$

from which we may identify an effective potential governing the wall position,

$$
2 \mathrm{U}(\mathrm{R})=1-\left(\frac{\mathrm{R}}{\gamma}+\kappa_{2} \frac{\gamma^{2}}{\mathrm{R}^{2}}\right)^{2}-\kappa_{1} \frac{\gamma}{\mathrm{R}}
$$

where we have introduced the parameters

$$
\gamma=\frac{4 \bar{\sigma} \ell^{2}}{4 \bar{\sigma}^{2} \ell^{2}+1}, \quad \kappa_{1}=\frac{2 G M_{-}}{\gamma}, \quad \kappa_{2}=\frac{G \Delta M}{2 \bar{\sigma} \gamma^{2}} .
$$

It is clear that the overall qualitative nature of the solution depends only $\kappa_{1}$ and $\kappa_{2}$ with solutions at different values of $\gamma$ reached under simultaneous $\lambda$ and $R$ rescalings. Note however that the value of $\bar{\sigma}$ is important in determining $\dot{t}_{ \pm}$. The potential is qualitatively similar for varying $\kappa_{1}, \kappa_{2}$, and is illustrated in figure 2 for $\kappa_{1}=0$. For fixed $\kappa_{2}$, switching on $\kappa_{1}$ has a similar effect to increasing $\kappa_{2}$, in that the potential is lowered, and the range of disallowed $\tilde{R}=R / \gamma$ is decreased.

For any value of $\kappa_{2}$, there is a critical value of $\kappa_{1}$ for which the maximum of the potential is at zero, delineating bouncing solutions from transmission solutions. This critical value can be obtained by simultaneously solving $U=U^{\prime}=0$, and leads to a maximal value $\kappa_{1}^{*}\left(\kappa_{2}\right)$, derived in appendix B (eq. (B.2)). Physically, we are restricted to $\kappa_{1} \geq 0$, however, we see from appendix $\mathrm{B}$ that we can have $\kappa_{2}<0$, or a "bigger on the inside" mass with a regular bounce solution. The only constraint on $\kappa_{1,2}$ is that they allow for a range of $\tilde{R}$ for which $U$ is positive, determined by (B.1), and also that $\dot{t}_{ \pm} \geq 0$, which ensures we have a positive tension properly oriented wall, and determines a minimum value for $\kappa_{1}$ (eq. (B.4)). 


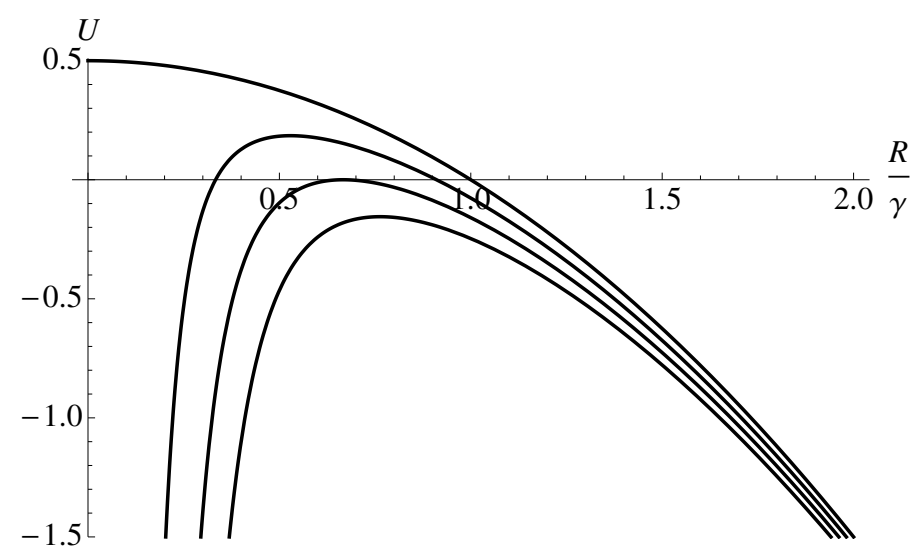

Figure 2. The potential $U$ shown here with $\kappa_{1}=0$. From top to bottom we show $\kappa_{2}=$ $0, \frac{1}{2} \kappa_{*}, \kappa_{*}, \frac{3}{2} \kappa_{*}$, where $\kappa_{*}=4 / 27$ marks the transition from bounce to transmission, with an unstable fixed- $r$ solution when $\kappa_{2}=\kappa_{*}$.

Because we will ultimately be interested in Euclidean wall trajectories, we shall consider only those bubble solutions which reflect. For certain parameters we can construct the solutions analytically. For example, at $\kappa_{1}=\kappa_{2}=0$ we have,

$$
\begin{aligned}
R(\lambda) & =|\gamma| \cosh \left(\frac{\lambda}{\gamma}\right) \\
t_{-}(\lambda) & =\gamma \sinh \left(\frac{\lambda}{\gamma}\right) \\
\sqrt{\ell^{2}-\gamma^{2}} \tanh \left(\frac{t_{+}(\lambda)}{\ell}\right) & =\gamma \sinh \left(\frac{\lambda}{\gamma}\right)
\end{aligned}
$$

which parametrically describes the trajectory, $\cosh ^{2}\left(\frac{t_{+}}{\ell}\right)\left(r^{2}-\ell^{2} \tanh ^{2}\left(\frac{t_{+}}{\ell}\right)\right)=\gamma^{2}$. To see that this solution is just the CDL solution, [10], but in the causal patch, we make the coordinate transformation $r(\rho, \chi), t_{+}(\rho, \chi)$, with

$$
\cosh ^{2}\left(\frac{t_{+}}{\ell}\right)\left(r^{2}-\ell^{2} \tanh ^{2}\left(\frac{t_{+}}{\ell}\right)\right)=\ell^{2} \sin ^{2}\left(\frac{\rho}{\ell}\right), \quad r^{2}=\ell^{2} \sin ^{2}\left(\frac{\rho}{\ell}\right) \sin ^{2} \chi
$$

which results in the metric on a round $\mathrm{S}^{4}$

$$
d s^{2}=d \rho^{2}+\ell^{2} \cos ^{2}\left(\frac{\rho}{\ell}\right)\left(d \chi^{2}+\sin ^{2} \chi d \Omega_{2}^{2}\right)
$$

with the bubble wall sitting at fixed $\rho$, specifically, $\ell^{2} \cosh ^{2}\left(\frac{\rho}{\ell}\right)=\gamma^{2}$, which agrees with [10]. ${ }^{1}$ Note however that $(2.12)$ requires $2 \bar{\sigma} \ell<1$ for $\dot{t}_{+} \geq 0$, in essence stating that the CDL bubble wall must remain within the static patch. Clearly one does not have this restriction in the original CDL approach, however, for $2 \bar{\sigma} \ell>1$, the bubble has now consumed more than half of the de Sitter hyperboloid.

\footnotetext{
${ }^{1}$ To compare with [10] we note some notational differences, we have $\bar{\rho}_{C D L}=\gamma, S_{1, C D L}=\sigma, \kappa_{C D L}=8 \pi G$ and $\varepsilon_{C D L}=3 /\left(8 \pi G \ell^{2}\right)$.
} 


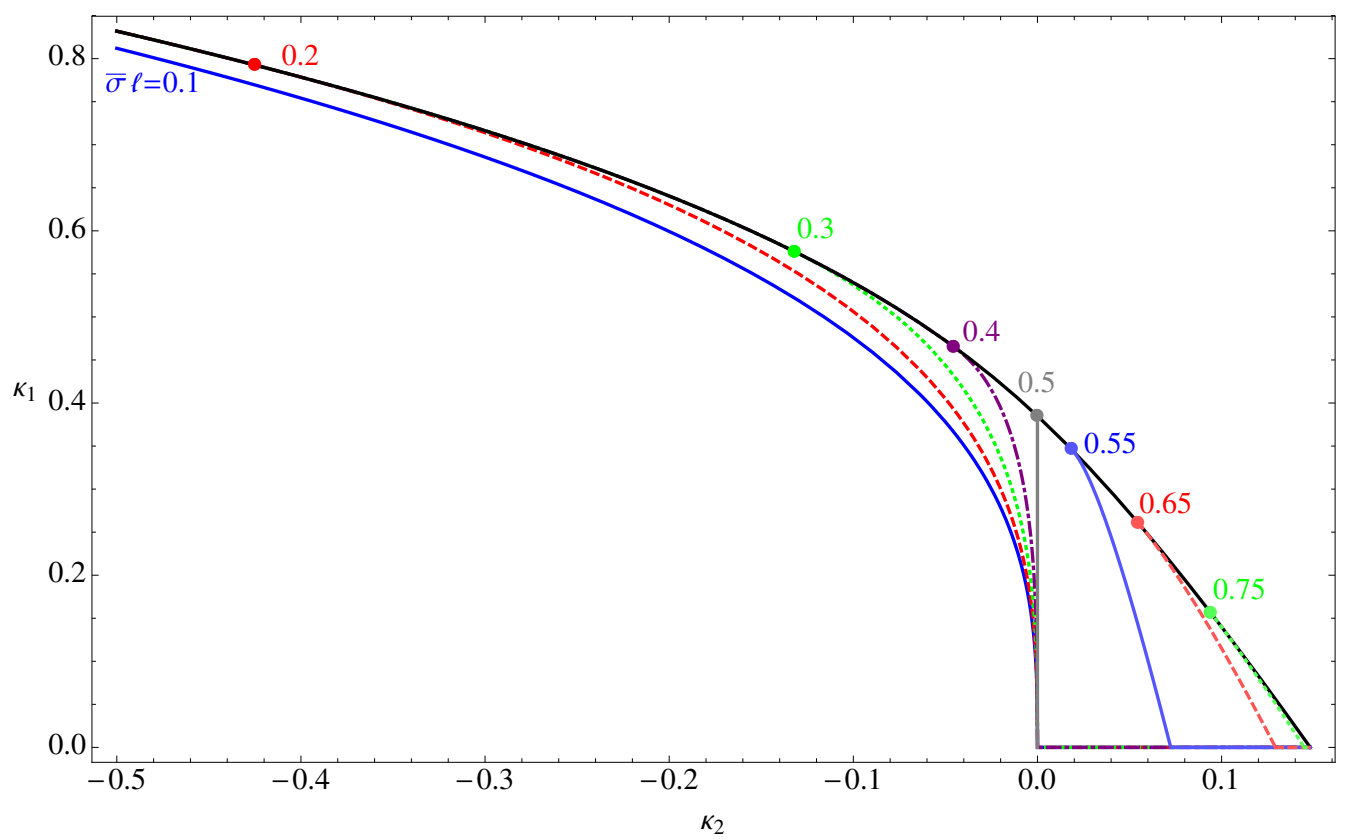

Figure 3. The allowed range of $\kappa_{2}$ and $\kappa_{2}$ for $\bar{\sigma} \ell \leq \sqrt{3} / 2$. The upper solid black line shows the maximum value of $\kappa_{1}, \kappa_{1}^{*}$, for a given $\kappa_{2}$. The lower lines show the minimum value allowed for $\kappa_{1}$ at various values of $\bar{\sigma} \ell$. From left to right: $\bar{\sigma} \ell=0.1$ in solid blue, 0.2 in dashed red, 0.3 in dotted green, 0.4 in dot-dash purple, 0.5 in grey, 0.55 in light blue, 0.65 in light dashed red, and 0.75 in light dotted green. The data points in matching color indicate the limiting value of $\kappa_{2}$ for each $\bar{\sigma} \ell$. The allowed range closes off entirely above $\bar{\sigma} \ell=\sqrt{3} / 2$.

A second family of analytic solutions can be found when $\kappa_{1}$ and $\kappa_{2}$ satisfy (B.1), $\kappa_{1}=\kappa_{1}^{*}\left(\kappa_{2}\right)$, for which there is a critical, unstable bubble wall solution sitting at the maximum of the potential, $R_{*}=2^{-2 / 3} \gamma\left(\kappa_{1}^{*}+2 \kappa_{2}+\sqrt{\kappa_{1}^{* 2}+4 \kappa_{1}^{*} \kappa_{2}+36 \kappa_{2}^{2}}\right)^{1 / 3}$. Finally, for the remaining solutions, ${ }^{2} 0<\kappa_{1}<\kappa_{1}^{*}$, we find that there are two solutions, corresponding to small- $r$ and large- $r$ bounces. The former corresponds to a bubble entering through the past black hole horizon, growing, turning around and then falling through the future black hole horizon, whilst the latter corresponds to the same process but for the cosmological horizon; this is easily seen from the potential. We have explicitly constructed examples of these trajectories numerically.

\subsection{Euclidean bubbles}

The effect of performing a Wick rotation $t=-i \tau$ can be described in the equations of the last section by simultaneously Wick rotating the world-volume proper time, $\lambda=-i x$. The overall effect of this on the equation governing the bubble wall position is a flip in the sign of the potential, (2.14). Thus we obtain solutions only in the parameter range for which Lorentzian bounce solutions exist.

In appendix B, we detail the constraints on $\kappa_{1}, \kappa_{2}$ for a regular instanton to exist. Briefly, for a bounce, we require $U \geq 0$ for some range of $\tilde{R}$, leading to an upper bound

\footnotetext{
${ }^{2}$ The range of $\kappa_{1}$ can be modified if $\kappa_{2}<0$, as we discuss in the next subsection, $\S 2.2$.
} 
$\kappa_{1} \leq \kappa_{1}^{*}$, given in (B.1). However, a regular instanton also requires positivity of $\dot{\tau}_{+}$, which leads to a lower bound $\kappa_{1} \geq \kappa_{1, m i n}$, as derived in (B.4). Figure 3 shows the allowed parameter ranges of $\kappa_{1}$ and $\kappa_{2}$ for a selection of values of $\bar{\sigma} \ell$. The range of $\kappa_{1}$ is shown as a function of $\kappa_{2}$, the maximum $\kappa_{1}^{*}$ being shown as a solid black line. For a selection of $\bar{\sigma} \ell$, the minimum value of $\kappa_{1}$ is shown, as well as the limiting value where the range of $\kappa_{1}$ eventually closes off, i.e.

$$
\kappa_{2}=\frac{-(1-2 \bar{\sigma} \gamma) \ell^{3}}{3 \sqrt{3} \gamma^{3}}, \quad \kappa_{1}=\frac{\left(3-4 \bar{\sigma}^{2} \ell^{2}\right) \ell}{3 \sqrt{3} \gamma} \Rightarrow G M_{+}=G M_{N}, \quad R_{*}=\frac{\ell}{\sqrt{3}}
$$

For $\kappa_{2} \leq-(1-2 \bar{\sigma} \gamma) \ell^{3} /\left(3 \sqrt{3} \gamma^{3}\right)$, there is no well defined solution, and it follows that there is an upper limit to the tension of $\bar{\sigma} \ell<\sqrt{3} / 2$. On the other hand, for any large negative $\kappa_{2}$ we can always find a $\bar{\sigma}$ small enough to allow for a bounce.

When $\kappa_{1, \min }<\kappa_{1}<\kappa_{1}^{*}$ there are a pair of Lorentzian bounce solutions, and correspondingly there is a single Euclidean bubble wall which is periodic in $x$ with the same turning points. Note that the periodicity in the time coordinates on each side of the Euclidean wall need not be, and indeed in general is not, the same. Again, we have constructed these solutions numerically.

When $\kappa_{1}=\kappa_{2}=0$ corresponding to the CDL case, there is only a single Lorentzian bounce solution. In the Euclidean picture the bubble begins at $R=0$ when $x=-\gamma \pi / 2$, grows to its maximum size at the turning point, and then retreats once more to $R=0$ when $x=\gamma \pi / 2$. This can be seen by Wick rotating the Lorentzian solution (2.16)-(2.18). Notice that this interval in the external Euclidean time coordinate, $\tau_{+}$, runs over a particular range, $\beta_{\mathrm{CDL}}$, which does not correspond to the regularity condition at the cosmological horizon, in general. We also note that $\beta_{\mathrm{CDL}}=\lim _{\kappa_{1}, \kappa_{2} \rightarrow 0} \Delta \tau_{+}$, so it is continuously connected to the massive oscillatory solutions in an appropriate sense.

\section{Computing the Euclidean action}

In the last section we computed Lorentzian and Euclidean thin-wall bubble trajectories. The Euclidean trajectories oscillate between the turning points of the corresponding Lorentzian solutions. In this section we compute the Euclidean on-shell action for the bubble-wall solution $I$, and for no bubble-wall $I_{S D S}$, from which we calculate

$$
\Gamma \propto e^{-B}, \quad \text { where } \quad B=I-I_{\mathrm{SdS}} .
$$

We claim that $\Gamma$ gives the rate at which bubbles are nucleated in a false vacuum SdS universe, centred on the black hole. Similar claims are made for calculation of the decay rate of the false vacuum in de Sitter space, [10], for black hole nucleation, [24-26], and for open universe nucleation, $[13,14]$. The only case where the formula has a rigorous justification is in flat space, [1]. An interesting interpretation of the CDL instanton with some support for the formula has been given in [27]. Nevertheless, the use of instantons to calculate nucleation rates in curved space has to be treated as speculative, and the results considered with a degree of caution. 


\subsection{General results}

It is instructive to first consider the case where the Euclidean space $\mathcal{M}$ has a Killing vector, $\partial_{\tau}$. This can occur in the absence of a bubble wall, or for a $\tau$-independent wall configuration. As previously noted, $\mathcal{M}$ will in general contain a finite number of conical singularities, we also allow for a general scalar field in the space-time, provided it satisfies the required background symmetries.

The contributions from the conical deficits are determined by isolating them within a small region around each, $\mathcal{B}_{i}=\left\{x^{\mu}:\left|r-r_{i}\right|<\mathcal{O}\left(\epsilon^{2}\right)\right\}$, smoothing out the conical deficit, performing our integral, then sending $\epsilon \rightarrow 0$. Although in general, one cannot regulate a co-dimension two $\delta$-function singularity in general relativity, [28], for the particular case of a product metric, the limit is well-defined, as the ambiguity occurs due to nontrivial physical content in the transverse components of the energy-momentum tensor, which are not present in the special case of the product metric. We therefore write the EinsteinHilbert action as $I=I_{\mathcal{M}-\mathcal{B}}+I_{\mathcal{B}}$, where

$$
\begin{aligned}
I_{\mathcal{M}-\mathcal{B}} & =-\frac{1}{16 \pi G} \int_{\mathcal{M}-\mathcal{B}} \mathcal{R}-\int_{\mathcal{M}-\mathcal{B}} \mathcal{L}_{m}(g, \phi)+\frac{1}{8 \pi G} \int_{\partial \mathcal{B}} K \\
I_{\mathcal{B}} & =-\frac{1}{16 \pi G} \int_{\mathcal{B}} \mathcal{R}+\frac{1}{8 \pi G} \int_{\partial \mathcal{B}} K
\end{aligned}
$$

where the appropriate Gibbons Hawking boundary terms have been added (with inward pointing normals) at each ball boundary.

To evaluate this on-shell we perform a foliation of $\mathcal{M}-\mathcal{B}$ with a family of surfaces $\Sigma_{\tau}$ (assuming the global topology permits), with $0<\tau<\beta$. For this foliation we introduce coordinates with lapse $N$ and shift functions $N^{i}$, as well as the induced metric ${ }^{(3)} g_{i j}$, its conjugate momentum $\pi^{i j}$, and conjugate momentum of the matter field, $\pi$. The leaves of the foliation have boundaries at the ends, and the canonical decomposition of such foliations has been investigated by Hawking and Horowitz, [29]. The first piece of our action becomes,

$$
I_{\mathcal{M}-\mathcal{B}}=\frac{1}{16 \pi G} \int_{0}^{\beta} d \tau\left[\int_{\Sigma_{\tau}}\left({ }^{(3)} \partial_{\tau} g_{i j} \pi^{i j}+\partial_{\tau} \phi \pi-N \mathcal{H}-N^{i} \mathcal{H}_{i}\right)-\int_{\partial \mathcal{B}_{\tau}} N k\right]
$$

where $\mathcal{H}$ and $\mathcal{H}_{i}$ are the Hamiltonian and momentum constraints and $k$ is the extrinsic curvature of $\partial \mathcal{B}_{\tau} \equiv \partial \mathcal{B} \cap \Sigma_{\tau}$. We have $\mathcal{H}=\mathcal{H}_{i}=0$ and furthermore the symmetry implies $\partial_{\tau} \phi={ }^{(3)} \partial_{\tau} g_{i j}=0$. The contribution from the Gibbons-Hawking term is sub-leading in the expansion about the conical singularity, and hence $N k=O(\epsilon)$, i.e. $I_{\mathcal{M}-\mathcal{B}}=0$ to leading order. This possibly surprising result is readily confirmed by a direct computation of the action $I_{\mathcal{M}-\mathcal{B}}$ in the case of a pure cosmological constant 'dark energy' source.

Turning to the contribution from the conical singularities, we show in the appendix that the contribution from a single conical defect region $B_{i}$ is given by its area $\mathcal{A}_{i}$,

$$
-\int_{B_{i}} \mathcal{R}+2 \int_{\partial B_{i}} K=-4 \pi \mathcal{A}_{i}
$$


Putting all the contributions to the action together gives

$$
I=-\frac{1}{4 G} \sum_{i} \mathcal{A}_{i}
$$

At first sight, this appears to be an example of the classic 'NUT's and bolts' formula for the action of a gravitational instanton due to Gibbons and Hawking, [15], but the difference is that we have extended the result to singular instantons. The remarkable feature is that the conical deficit angle does not appear in the action, which is explicitly independent of the period $\beta$. As a consequence, a possible source of ambiguity in using singular instantons has been annulled. We shall employ this result in order to compute the Euclidean on-shell action for SdS and static bubbles, although the result applies in any dimension, and can be further extended to rotating or charged spaces without any difficulty.

Solutions with a moving bubble wall break the time-translation symmetry of the full space-time, but the result can be extended if the geometries on both sides of the bubble wall still individually possess the Killing vector, $\partial_{\tau}$. Consider the spherically symmetric metric

$$
d s^{2}=f(r) d \tau^{2}+f(r)^{-1} d r^{2}+r^{2} d \Omega^{2},
$$

where now we allow two conical defects at $r_{h}$ and $r_{c}$, and (in principle) a more general form for $f(r)$ than used previously. There is a wall whose location is parametrised by $r=R(\lambda)$ and $\tau(\lambda)$, as illustrated in figure 1 . Let $\mathcal{M}_{ \pm}$denote the regular parts of the manifold to the right and left of the wall, regions $\mathcal{B}$ covering the conical defects as before, and $\mathcal{W}$ the contribution of the wall itself and split the action into contributions from each region,

$$
I=I_{\mathcal{B}}+I_{-}+I_{+}+I_{\mathcal{W}}
$$

where $\mathcal{B}$ covers the conical defects as before,

$$
I_{\mathcal{W}}=-\int_{\mathcal{W}} \mathcal{L}_{m}(g, \phi)=\int_{\mathcal{W}} \sigma
$$

is the action of the thin wall, and

$$
I_{ \pm}=-\frac{1}{16 \pi G} \int_{\mathcal{M}_{ \pm}} \mathcal{R}-\int_{\mathcal{M}_{ \pm}} \mathcal{L}_{m}(g, \phi)+\frac{1}{8 \pi G} \int_{\partial \mathcal{M}_{ \pm}} K
$$

are the remaining bulk actions with the relevant Gibbons-Hawking boundary terms. Note $\partial \mathcal{M}_{ \pm}$include both the boundaries at the conical deficit excision balls, as well as the boundary on each side of the wall. As is conventional, these boundary integrals are evaluated with inward pointing normals, which means that on the inner wall boundary $r_{-}=R$, this normal will in fact have the opposite sign to the one usually used in the computation of the Israel junction conditions, and therefore there will be an apparent sign difference when we come to use that substitution, which is simply due to this vexatious disparity in conventions.

In order to decompose the action into space and Euclidean time we use the identity

$$
\mathcal{R}={ }^{3} \mathcal{R}-K^{2}+K_{a b}^{2}-2 \nabla_{a}\left(u^{a} \nabla_{b} u^{b}\right)+2 \nabla_{b}\left(u^{a} \nabla_{a} u^{b}\right),
$$


where the vector $u^{\mu}$ is normal to $\Sigma_{\tau}[29]$. After integration by parts, and taking the conical deficit excision radius $\epsilon \rightarrow 0$, we obtain

$$
\begin{aligned}
I_{ \pm}= & -\frac{1}{16 \pi G} \int_{0}^{\beta} d \tau \int_{\Sigma_{\tau}}\left({ }^{3} \mathcal{R}-K^{2}+K_{a b}^{2}-16 \pi G \mathcal{L}_{m}\right) \\
& -\frac{1}{8 \pi G} \int_{\mathcal{W}} K_{ \pm}+\frac{1}{8 \pi G} \int_{\mathcal{W}} n_{ \pm b} u^{a} \nabla_{a} u^{b}
\end{aligned}
$$

with $n_{ \pm} a= \pm(\dot{\tau} d r-\dot{r} d \tau)$ the inward pointing one-form normal to $\mathcal{W}$ as described above. The first integral reproduces the canonical action we had previously, and vanishes due to the killing symmetry and the constraints. The second term represents the integration of the singular part of the Ricci scalar due to the thin wall, and by Israel's junction condition (remembering the sign disparity) the extrinsic curvatures on each side of the wall are related via $K_{+}=-4 \pi G S-K_{-}=12 \pi G \sigma-K_{-}$for the surface stress tensor $S_{a b}=-\sigma h_{a b}$. The final contribution is a boundary term coming from the wall, and $u^{a} \nabla_{a} u^{b} \partial_{b}=-f^{\prime} \partial_{r} / 2$ giving,

$$
I_{ \pm}=\mp \frac{1}{16 \pi G} \int_{\mathcal{W}} f_{ \pm}^{\prime} \dot{\tau}_{ \pm}
$$

where the integrand is evaluated using the metric components on the appropriate side of the wall.

Pulling all these pieces together with the previous result for the conical defects we reach our final result,

$$
I=-\frac{1}{4 G}\left(\mathcal{A}_{h}+\mathcal{A}_{c}\right)-\frac{1}{2} \int_{\mathcal{W}} \sigma-\frac{1}{16 \pi G} \int_{\mathcal{W}}\left(f_{+}^{\prime} \dot{\tau}_{+}-f_{-}^{\prime} \dot{\tau}_{-}\right)
$$

for the action of a space-time with a bubble wall separating two regions of possibly different black hole masses and effective cosmological constants.

\section{Tunnelling from the false vacuum}

Having demonstrated how to calculate the action of a singular instanton, we would like to apply the method to the situation of tunnelling catalysed by a "point source impurity" the black hole. In general we can consider the case where a remnant black hole remains in the true vacuum after the nucleation process, possibly with a different mass from the original black hole. We shall consider the general case in section 4.4. First however, we consider some special cases, where the seed black hole is wiped out during the tunnelling process, leaving no remnant black hole within the interior of the bubble, or where the remnant black hole corresponds to the 'static' bounce.

For future reference, in the case of tunnelling from SdS to general Schwarzschild $\left(\Lambda_{-}=\right.$ $0, \Lambda_{+}=\Lambda$, general $M_{+}, M_{-}$), we note the expressions for the (Euclidean) wall trajectory

$$
\dot{R}^{2}=1-\frac{2 G M_{-}}{R}-\left(\frac{R}{\gamma}+\frac{G \Delta M}{2 \bar{\sigma} R^{2}}\right)^{2}
$$

and the action

$$
I_{\kappa_{1}, \kappa_{2}}=\frac{1}{4 G}\left[-\left(\mathcal{A}_{h}+\mathcal{A}_{c}\right)+\int d \lambda\left[\left(2 R-6 G M_{+}\right) \dot{\tau}_{+}-\left(2 R-6 G M_{-}\right) \dot{\tau}_{-}\right]\right]
$$


where we have used the euclidean Israel equation to substitute for $\bar{\sigma}$, and for clarity of presentation we have labelled the action using subscripts for the parameters $\kappa_{1}$ and $\kappa_{2}$. We consider the two analytic cases first, then discuss other cases numerically.

\subsection{Coleman de Luccia bubbles}

The first case we consider in the nucleation of a Minkowski region in de Sitter space through the CDL process, where the masses $M_{+}=M_{-}=0$. Recall that the Lorentzian CDL bubble in the static patch is parameterised by (2.16)-(2.18), which gives the Euclidean bubble:

$$
R=\gamma \cos \left(\frac{\lambda}{\gamma}\right), t_{-}=\gamma \sin \left(\frac{\lambda}{\gamma}\right), t_{+}=\ell \arctan \left[\frac{\gamma \sin \left(\frac{\lambda}{\gamma}\right)}{\sqrt{\ell^{2}-\gamma^{2}}}\right]
$$

Now, we take the integration in (4.2) to cover a single interval for which $R>0$, i.e. the wall traverses the interval $-\gamma \pi / 2 \leq \lambda \leq \gamma \pi / 2$. Notice that this interval in the external Euclidean time coordinate, $\tau_{+}$, runs over a particular range, $\beta_{+}$, which does not correspond to the regularity condition at the cosmological horizon in general.

Inputting the functions (4.3) into (4.2) gives

$$
\int d \lambda R\left(\dot{\tau}_{+}-\dot{\tau}_{-}\right)=\pi\left[2 \ell^{2}-\gamma^{2}-2 \ell \sqrt{\ell^{2}-\gamma^{2}}\right]
$$

requiring tensions $4 \bar{\sigma}^{2} \ell^{2}<1$. We also have $\mathcal{A}_{h}=0$ and $\mathcal{A}_{c}=4 \pi \ell^{2}$, leaving us with

$$
I_{0,0}=-\frac{\pi \ell^{2}}{G} \frac{1+8 \bar{\sigma}^{2} \ell^{2}}{\left(1+4 \bar{\sigma}^{2} \ell^{2}\right)^{2}}
$$

The exponent $B=B_{\mathrm{CDL}}$ and the associated tunnelling rate can then be obtained simply from (3.1), with $I_{\mathrm{SdS}}=I_{\mathrm{dS}}$ in this case,

$$
B_{\mathrm{CDL}}=\frac{\pi \ell^{2}}{G} \frac{16 \bar{\sigma}^{4} \ell^{4}}{\left(1+4 \bar{\sigma}^{2} \ell^{2}\right)^{2}}
$$

in agreement with the non-singular instanton calculation, [10].

\subsection{The critical static bubble wall with $M_{-}=0$}

The second analytic solution we considered in section 2 was the critical case with $\kappa_{1}=$ $\kappa_{1}^{*}\left(\kappa_{2}\right)$. When $M_{-}=0$, this corresponds to the special choice $\kappa_{2}=\kappa_{*}=4 / 27$ which is when there exists a single unstable Lorentzian bubble wall at fixed radius $R=2 \gamma / 3$. We may calculate the action using the integrals in (4.2), where the result is independent of the integration range, or we may simply use the result (3.6) to obtain in both cases,

$$
I_{0, \kappa_{*}}=-\frac{\pi r_{c}^{2}}{G} .
$$

The exponent $B$ and the tunnelling rate can be obtained simply from (3.1), specialising $I_{\mathrm{SdS}}$ to $\kappa_{2}=\kappa_{*}$ space-times. We find that in this case,

$$
B_{*}=\frac{\pi r_{h}^{2}}{G}
$$

Significantly, we find that $B_{*}<B_{\mathrm{CDL}}$ when compared at the same tension, $\bar{\sigma}$. 


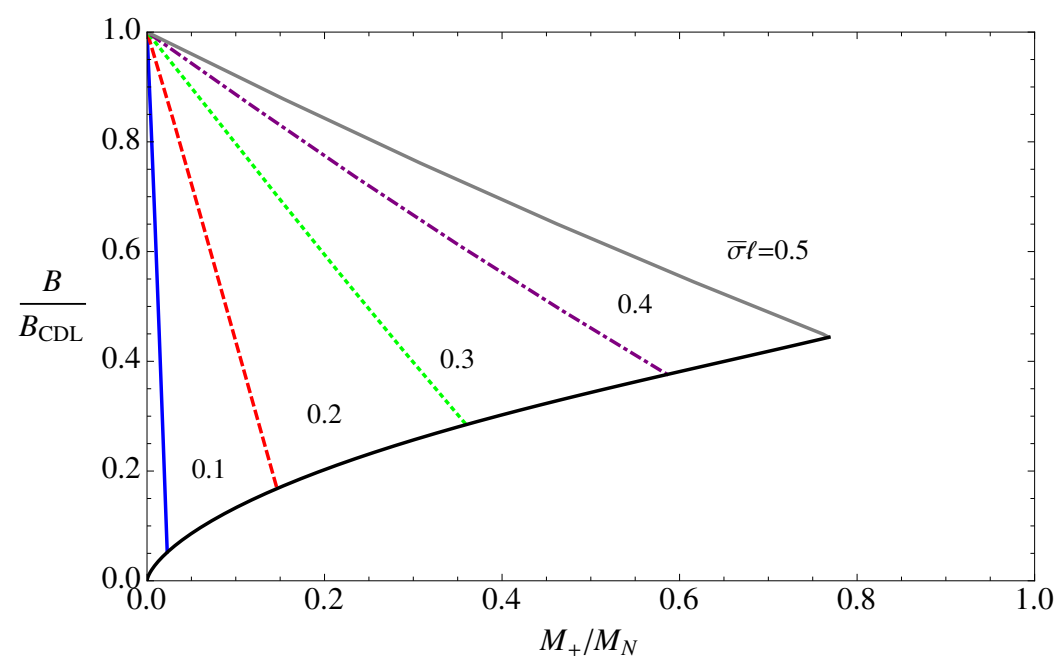

Figure 4. The decay rate exponent $B$ for a Minkowski bubble, as given by (3.1), as a function of the seed mass $M_{+}$for fixed tension $\bar{\sigma} \ell$. The values are scaled by $B_{C D L}$, the value of $B$ for the CDL instanton, and the Nariai mass $M_{N}=\ell / \sqrt{27} G$. From left to right we have $\bar{\sigma} \ell=0.1$ in solid blue, $\bar{\sigma} \ell=0.2$ in dashed red, $\bar{\sigma} \ell=0.3$ in dotted green, $\bar{\sigma} \ell=0.4$ in dot-dash purple, and $\bar{\sigma} \ell=0.5$ in grey. The lower solid black curve indicates the 'static' solutions, $\kappa_{2}=\kappa_{*}$, which fixes a relationship between mass and tension. In all cases, $B<B_{C D L}$ indicating that the decay rate is higher for a finite mass, at fixed tension.

\subsection{General $M_{-}=0$ bubbles}

Away from the special cases discussed above we must solve the wall trajectory equations (4.1) and (2.12) numerically. As with the CDL case, when evaluating the integrals in the action (4.2), we integrate over one period of the Euclidean wall trajectory. The instanton actions computed, $I$, are used to evaluate the exponent $B$ in the decay rate (3.1). We then compare $B$ with its $M=0$ value, $B_{\mathrm{CDL}}$, at fixed tension. A sample of results are shown in figure 4 . In all cases with finite $M$, as with the critical bubble wall case in section 4.2 , we find

$$
B<B_{\mathrm{CDL}}
$$

when compared at fixed tension, $\bar{\sigma} \ell$. In particular, we observe that $B$ is a monotonically decreasing function of $M$ at fixed $\bar{\sigma} \ell$, hence the nucleation rate is monotonically increasing with the mass of the black hole, $M$.

Figure 4 also shows that that there is no lower limit to the ratio $B / B_{C D L}$. This is in contrast to the results of Hiscock [5], who gave an lower bound of around 0.6. The difference is due to the fact that Hiscock did not include the contribution to the action from the conical singularities.

\subsection{The critical static bubble wall with $M_{-} \neq 0$}

The simplest situation in which a black hole remains behind after the tunnelling is the critical case where the masses are determined by the value of $\bar{\sigma} \ell$ and the condition $\kappa_{1}=$ $\kappa_{1}^{*}\left(\kappa_{2}\right)$. For this critical bubble, we must solve the constraints (B.1), then determine the 
decay rate exponent for the 'static' bounce, which is given by

$$
B_{*}=\frac{\pi\left(r_{h}^{2}-\left(2 G M_{-}\right)^{2}\right)}{G} .
$$

Although all the expressions for $\kappa_{1}^{*}, r_{h}\left(G M_{+}\right)$are algebraic, their form is not particularly illuminating (though we present them in an appendix for completeness). We find numerically that the bounce action is always positive and minimal when $\kappa_{2}$ is maximal, for all values of $\bar{\sigma} \ell$. Naively, we might expect from (4.10) that large remnant masses would have the smallest values of $B_{*}$. Paradoxically, we find that tardis like solutions, where the mass is bigger on the inside, generally have larger action than the CDL case.

\subsection{The dominant processes}

In the previous subsections 4.1-4.4, we have detailed the behaviour of the Euclidean instanton actions in various special cases. The generic bubble solution depends on the masses $M_{+}$and $M_{-}$as well as the tension parameter $\bar{\sigma} \ell$, and the action has to be evaluated numerically. The important question is which one of these bubble solutions represents the dominant physical process. Specifically, for a given seed black hole mass, $M_{+}$, we wish to minimise $B / B_{C D L}$ with respect to the remnant mass, $M_{-}$.

We have not been able to prove analytically which bubble solutions give the dominant rate, though we are able to perform a comprehensive numerical investigation. Here, we present results at a single fixed tension, $\bar{\sigma} \ell=0.2$, though the other cases are qualitatively the same. For these bubbles, the quantity $B$ is shown in figure 5 for a selection of remnant masses $M_{-}$over the full range of $M_{+}$. Clearly, where they exist, the static bubbles provide the dominant contribution at fixed $M_{+}$, and where they do not, the $M_{-}=0$ solutions dominate. These solutions are indicated by the dashed lines in the figure. These are the solutions which maximise the mass difference, $\kappa_{2}$.

Figure 5 also indicates that the seed black hole which would give the highest rate process is given by a critical value, $M_{C}$ where,

$$
G M_{C}=\frac{8}{27} \bar{\sigma} \gamma^{2}
$$

as quoted earlier in (1.2). Extending the analysis to other tensions, we plot the value of $M_{-}$which maximises the rate at a given $M_{+}$in figure 6 , and the corresponding values of $B$ in figure 7. Consistent bubble solutions only exist when $\bar{\sigma} \ell<\sqrt{3} / 2$ (see figure 3).

The underlying reason for the existence of this critical value is that the static walls only exist when $M_{+} \geq M_{C}$. Correspondingly, when $M_{+}>M_{C}$ the dominant process is the nucleation of a static bubble wall solution as discussed in section 4.4. In this range the nucleation involves the creation of a black hole of finite mass $M_{-}$, indicated in figure 6 . For $M_{+}>M_{C}$ the rate is therefore given by $B_{*}$ in (4.10).

When $M_{+}<M_{C}$, there is no longer a static wall solution and the maximum $\kappa_{2}$ solution has no black hole remnant, $M_{-}=0$. These solutions were previously explored in section 4.3 and $B$-values shown in figure 4 . 


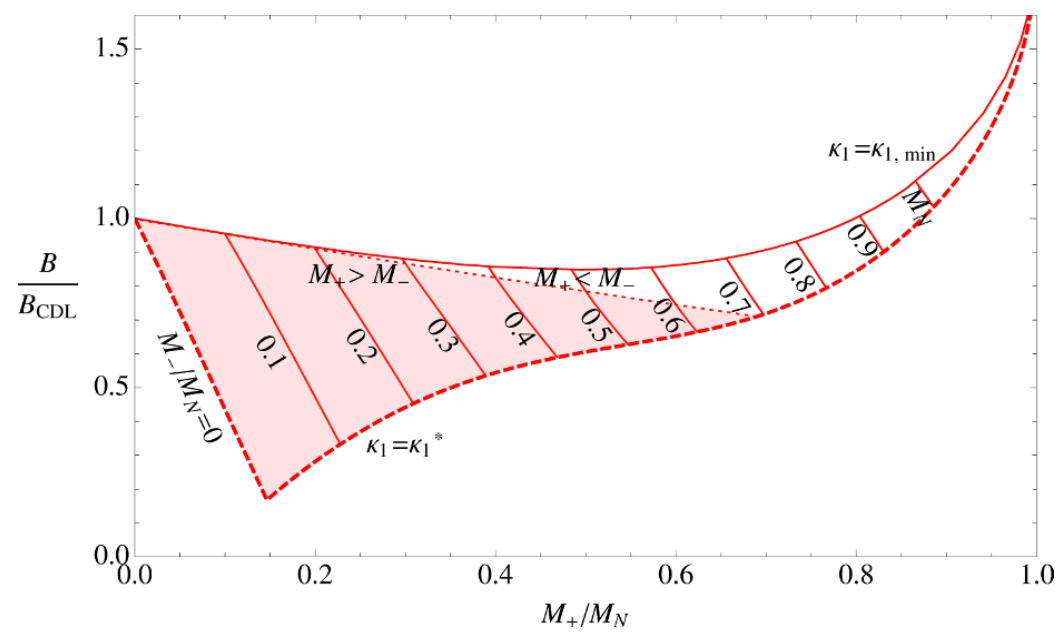

Figure 5. A scan of the full parameter space of allowed Euclidean instantons at fixed tension $\bar{\sigma} \ell=0.2$. The upper red solid curve, together with the dashed lines give the full envelope of allowed solutions at this tension. Also indicated are lines of constant remnant mass, $M_{-}$, showing that the dominant solutions are given by $M_{-}=0$ where they exist i.e. $M_{+}<M_{C}$ with $M_{C}$ defined in (4.11). For $M_{+}>M_{C}$ this data shows that the dominant solutions are the static walls indicated by $\kappa_{1}=\kappa_{1}^{*}$. The red shaded region corresponds to solutions where $M_{-}<M_{+}$, showing that for large enough $M_{+}$the dominant processes become tardis-like.

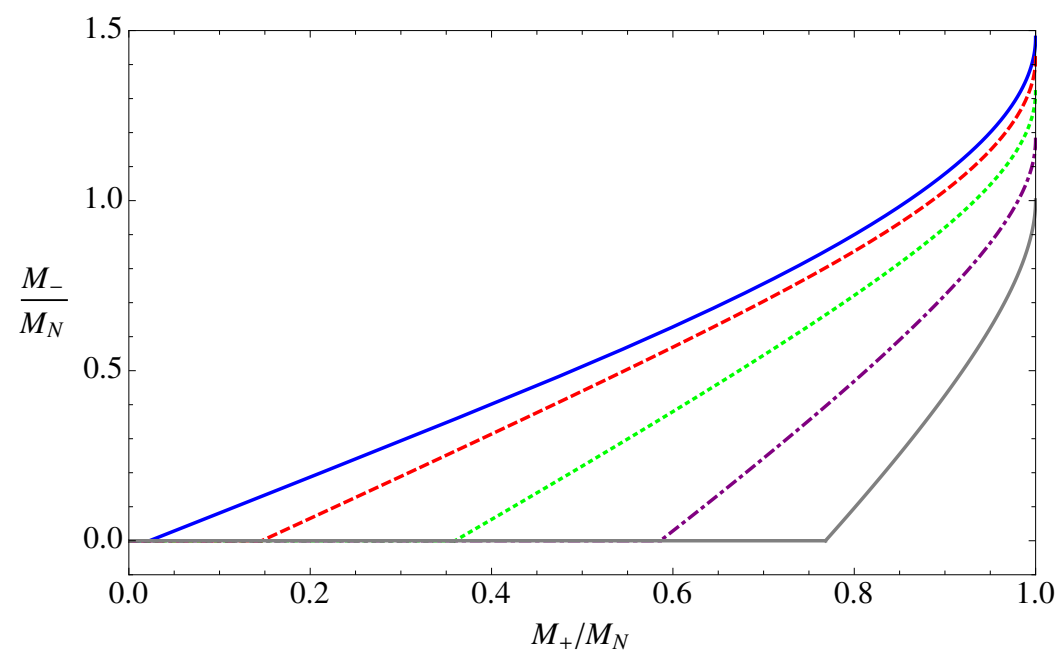

Figure 6. The remnant mass $M_{-}$as a function of the initial seed mass, $M_{+}$with fixed values of the tension $\bar{\sigma} \ell$. The remnant mass is only non-zero for sufficiently large seeds, $M_{+}>M_{C}$. The results for $\bar{\sigma} \ell=0.1$ are shown in solid blue, $\bar{\sigma} \ell=0.2$ in dashed red, $\bar{\sigma} \ell=0.3$ in dotted green, $\bar{\sigma} \ell=0.4$ in dot-dash purple, and $\bar{\sigma} \ell=0.5$ in gray.

\section{The WKB approach}

The WKB approach was introduced by Fischler et al., [18, 19], to calculate the probability for transitions between various thin-walled bubble solutions. In this section we shall compare the WKB approach to the singular instanton approach we have been describing. The 


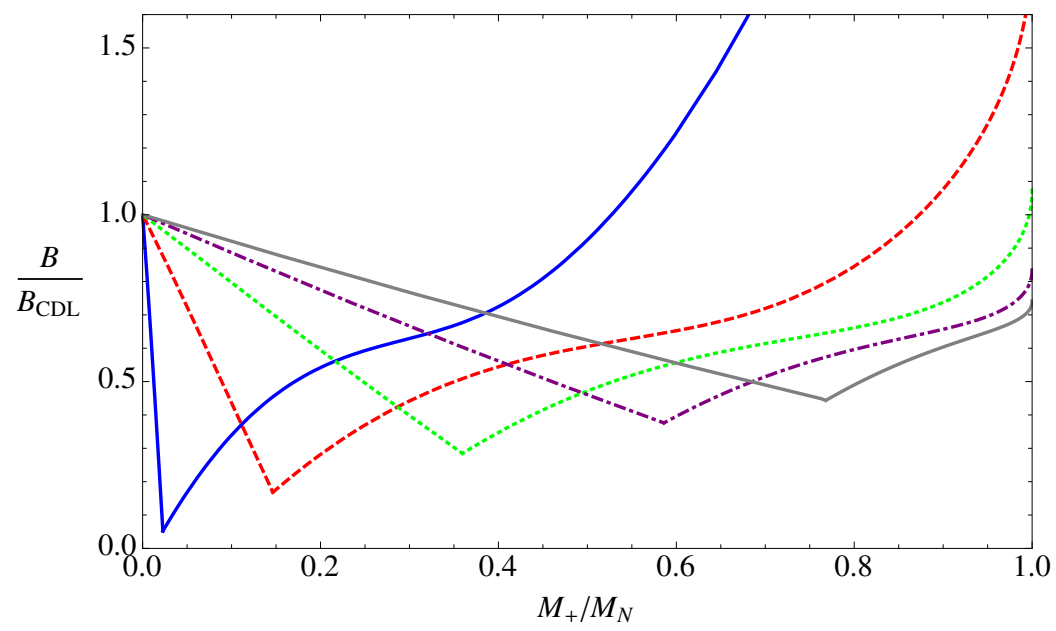

Figure 7. The exponent in the decay rate for the dominant decay process as a function of the initial seed mass $M_{+}$with fixed values of the tension $\bar{\sigma} \ell$. The value of $B$ is scaled by the CDL value and the mass by the Nariai mass $M_{N}=\ell / \sqrt{27} G$. The decay rate reaches its maximum value for the case of critical seed black holes $M_{+}=M_{C}$, as given by (4.11). The results for $\bar{\sigma} \ell=0.1$ are shown in solid blue, $\bar{\sigma} \ell=0.2$ in dashed red, $\bar{\sigma} \ell=0.3$ in dotted green, $\bar{\sigma} \ell=0.4$ in dot-dash purple, and $\bar{\sigma} \ell=0.5$ in gray.

basic idea of the WKB method is to formulate an action which depends only on the bubble wall trajectory and then use the associated Schrödinger equation to calculate tunnelling probabilities. Fischler et al. began with the Einstein-matter action and used a coordinate system adapted to the bubble wall. We introduce a far simpler version which uses the original static patch coordinates.

The gravity-matter action can be constructed in the same way as the Euclidean action discussion in section 3. The bubble wall is given by specifying an arbitrary function $R(\lambda)$. The background metric outside of the bubble wall is given by the same metric used earlier (3.7), and as usual with spherically symmetric metrics, the time-independence of the functions $f_{ \pm}(r)$ is a consequence of the constraint equations and independent of the wall trajectory. See [23] for a fuller discussion of this generalisation of Birkhoff's theorem, although note the caveats of $[32,33]$ when additional matter is present. Repeating the steps (3.7)-(3.14) in Lorentzian signature gives

$$
S=\frac{1}{16 \pi G} \int_{W}\left[f^{-1} f_{, r} \rho\right]_{-}^{+}+\frac{1}{8 \pi G} \int_{W}[K]_{-}^{+}-\int_{W} \sigma
$$

where $W$ is the bubble wall and

$$
\rho_{ \pm}=\left(f_{ \pm}+\dot{R}^{2}\right)^{1 / 2}
$$

The junction conditions determine the wall trajectory, and cannot be used at this stage, as we are seeking an effective action for this motion. Instead, we work off-shell and evaluate the trace of extrinsic curvature using the earlier results,

$$
K=\rho^{-1} \ddot{R}+\frac{1}{2} f_{, r} \rho^{-1}+2 R^{-1} \rho .
$$


After substituting the extrinsic curvature, the action can be written in terms of the Lagrangian of a one-dimensional dynamical system with coordinate $R(\lambda)$,

$$
S=\int L d \lambda
$$

where

$$
L=\frac{1}{2 G}\left[\rho^{-1} \ddot{R}+\frac{1}{2} f^{-1} f_{, r} \rho^{-1} \dot{R}^{2}+2 \frac{\rho}{R}\right]_{-}^{+}-4 \pi \sigma R^{2} .
$$

The classical equations of motion derived from this Lagrangian are second order but there is also a first order constraint which corresponds to relabelling of the coordinate $\lambda$ along the bubble wall. If we set $d \lambda=N d \lambda^{\prime}$ and then vary the action with respect to $N$, we find that the constraint reduces to the familiar junction condition ${ }^{3}$

$$
[\rho]_{-}^{+}=4 \pi G \sigma R .
$$

As before, we may rewrite the constraint as a conservation law,

$$
\frac{1}{2} \dot{R}^{2}+U=0
$$

In the quantum theory, this constraint becomes an operator $\mathcal{H}$ and the operator constraint $\mathcal{H} \Psi=0$ acting on the wave function $\Psi$ becomes the Schrödinger equation. Solutions can now tunnel through the barrier in the potential $V$. If we denote the tunnelling rate by $\Gamma_{b \rightarrow b}$, then the WKB approximation gives,

$$
\Gamma_{b \rightarrow b}=e^{i S\left[R_{b}\right]}
$$

where in this expression and in what follows the pre-factor to the exponential has been discarded. $R_{b}$ is the solution to the classical constraint with the complex 'time' parameter $\lambda \rightarrow i \lambda$. If we compare this to the instanton action $I$ evaluated earlier, by re-substituting $[K]$ back into the action, we notice that the contributions from the fixed points $r_{h}$ and $r_{c}$ are absent but otherwise $S$ is identical to $i I$. Consequently,

$$
\Gamma_{b \rightarrow b}=e^{-I-\left(\mathcal{A}_{-} / 4 G\right)-\left(\mathcal{A}_{c} / 4 G\right)},
$$

where $\mathcal{A}_{-}$is the area of the remnant black hole inside the bubble. We stress again that this represents the tunnelling rate from bubble solutions to other bubble solutions. However, we can compare this to the rate of false vaccum decay calculated using the instanton method,

$$
\Gamma=e^{-\left(I-I_{S d S}\right)}=e^{-I-\left(\mathcal{A}_{+} / 4 G\right)-\left(\mathcal{A}_{c} / 4 G\right)},
$$

where $\mathcal{A}_{+}$is the area of the seed black hole nucleating the bubble.

On the fully extended SdS spacetime, the bubble wall is replicated in different regions of the Penrose diagram, and so we might think of this as the rate for the spontaneous production of two bubbles from the false vacuum $\Gamma_{f \rightarrow b b}$, as illustrated in figure 8. Consequently, we have a relation of the form

$$
\Gamma_{f \rightarrow b b}=e^{-\left(\mathcal{A}_{+}-\mathcal{A}_{-}\right) / 4 G} \Gamma_{b \rightarrow b} .
$$

We interpret this as a type of crossing relation between spontaeous production of bubbles and bubble transitions, with the relative factor depending only on the black hole entropies.

\footnotetext{
${ }^{3}$ The orders of the field equation and the constraint are not obvious from an inspection of the Lagrangian.
} 

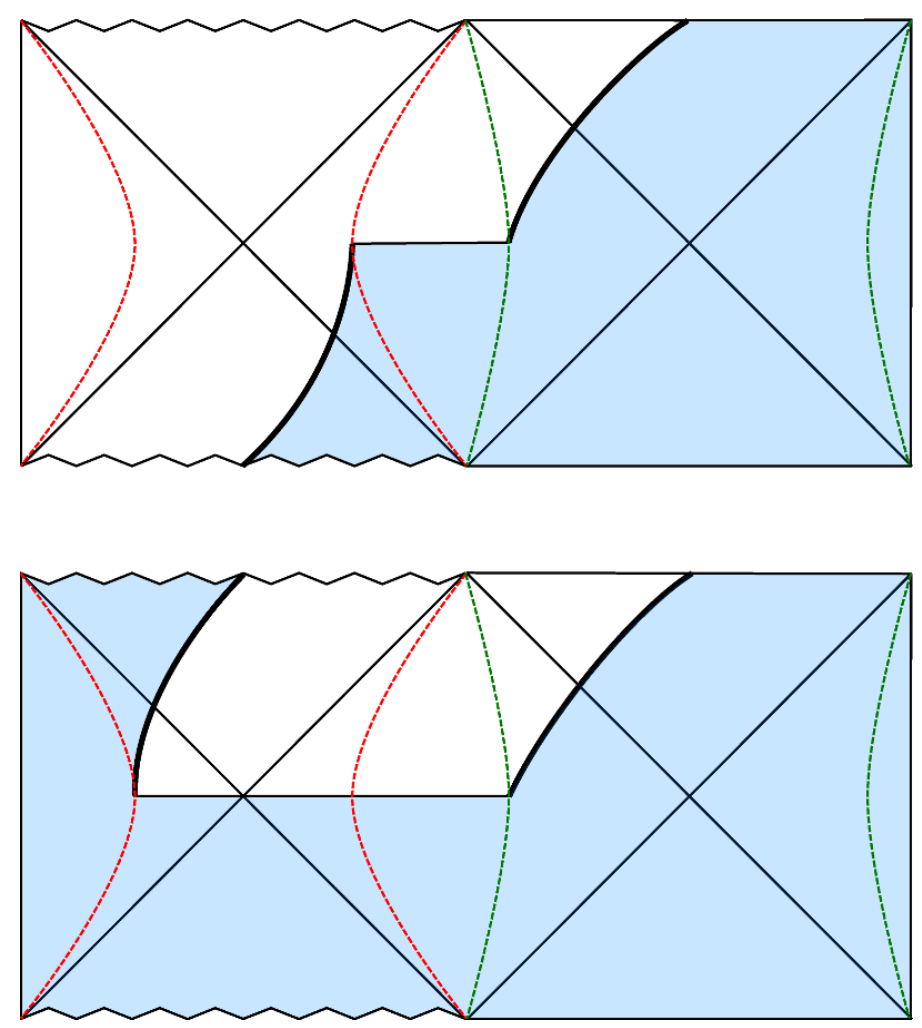

Figure 8. These Penrose diagrams showing pieces of SdS illustrate the the similarities between bubble-bubble transitions and bubble nucleations. The shaded regions represent the false vacuum. The bubble interiors are in the true vacuum with vanishing cosmological constant, and are not shown in the figures. In the first figure, the bubble wall starts out at $r=0$, grows to $r=r_{1}$, tunnels to $r_{2}$ and grows again to $r=\infty$. This is interpreted as a bubble $\rightarrow$ bubble transition. In the second figure, two bubble walls are spawned from the vacuum, corresponding to vacuum $\rightarrow$ bubble + bubble.

\section{Discussion}

In this paper we have explored the gravitational effect of inhomogeneities on false vacuum decay. Our main result is that the presence of the inhomogeneity generically acts to enhance the rate of true-vacuum bubble nucleation. The exception occurs only for seed black holes which approach the Nariai limit. The enhanced nucleation rates in the presence of a black hole could rescue some particle models which might otherwise get stuck in an early metastable state and never be able to decay into a radiation dominated universe.

We constructed a one-parameter family of Euclidean instantons at each fixed seed mass $M_{+}$. In general, the nucleation processes these instantons describe occur at a rate far higher than that of the $M_{+}=0$ or CDL process. In particular, for the limit of small seed masses $M_{+} \ll M_{N}$ and small tensions $\bar{\sigma} \ell \ll 1$, the decay exponent $B / B_{C D L}$ can be brought arbitrarily close to zero. The dominant decay at a fixed tension $\bar{\sigma} \ell$ depends on whether or not $M_{+}$exceeds a critical value, $M_{C}$. For $M_{+}>M_{C}$ the decay also nucleates a remnant black hole inside the vacuum bubble. For $M_{+}<M_{C}$ the decay simply nucleates the vacuum bubble with a flat interior. 
The salient technical point of our analysis is the consistent treatment of conical singularities in the Euclidean instanton calculation, ${ }^{4}$ which faithfully reproduce existing results which have been obtained using manifestly regular constructions. For instance, in section 4.1 we presented a derivation of the CDL instanton action starting from the Euclidean continuation of a single causal patch of de Sitter, containing conical singularities.

For the massless case $M_{-}=M_{+}=0$, and the critical mass case $\kappa_{2}=\kappa_{2}^{*}$ with $M_{-}=0$, equivalent regular instanton constructions are known. In these two cases it seems that the treatment of conical singularities we have employed can be considered a proxy for the existence of a regular construction in a different Euclidean section. It would be interesting to investigate whether this is the case in general, i.e., whether there is a family of regular instantons for the general case of $\kappa_{1}, \kappa_{2}$.

We have also found that the bubble nucleation rate calculated by the instanton approach is related to the bubble-to-bubble transition rate obtained in a Lorentzian WKB approach. One interpretation of this result is that the Euclidean instanton calculation actually describes the production of two true-vacuum bubbles in the fully extended Schwarzshild-de Sitter spacetime, only one of which is present in a single static patch. The connection with the bubble-to-bubble transition rate can then be explained as a type of crossing relation between bubble-to-bubble and the production of two bubbles from the false vacuum.

In this analysis we employed the simplest nucleation seed - a black hole. This may be introduced as an approximation to a spherical lump of matter, as shown by $S$ in figure 9 . The space-time outside of the matter is made up of a finite number of the regions from the fully extended SdS space-time. There is no longer any need to include a black hole at the antipodal point of the universe, and only one bubble nucleates around the lump of matter. Furthermore, the black hole approximation fails in a small region around the horizon, thus avoiding problems which would otherwise arise in defining stationary quantum states on the SdS background.

An important omission in this paper has been the neglect of Hawking radiation which may cause the black hole to evaporate before the vacuum decay can occur. Some idea of the relative decay rates can be obtained for black holes which are small compared to the horizon size. The largest enhancement of the vacuum decay rate occurs for a black hole with both $M_{-}=0$ and $M_{+}$at the critical value, $M_{C}$, when the decay rate is

$$
\Gamma_{*}=A_{*} e^{-B_{*}},
$$

where we have included the pre-factor $A_{*}$. From the results of section 4.2 we have $B_{*}=$ $4 \pi G M_{C}^{2}$. According to Callan and Coleman, [2], this pre-factor is made up from a factor of $\left(B_{*} / 2 \pi\right)^{1 / 2}$ for each translational zero mode of the instanton and a determinant factor. In our case, there will be a single zero mode representing the time translation symmetry. Rather than evaluate the determinant factor, we use the inverse horizon timescale as a rough estimate $\left(G M_{C}\right)^{-1}$, then

$$
\Gamma_{*} \approx\left(\frac{2}{G}\right)^{1 / 2} e^{-4 \pi G M_{C}^{2}}
$$

\footnotetext{
${ }^{4}$ These singularities were not taken into account in previous work [5].
} 


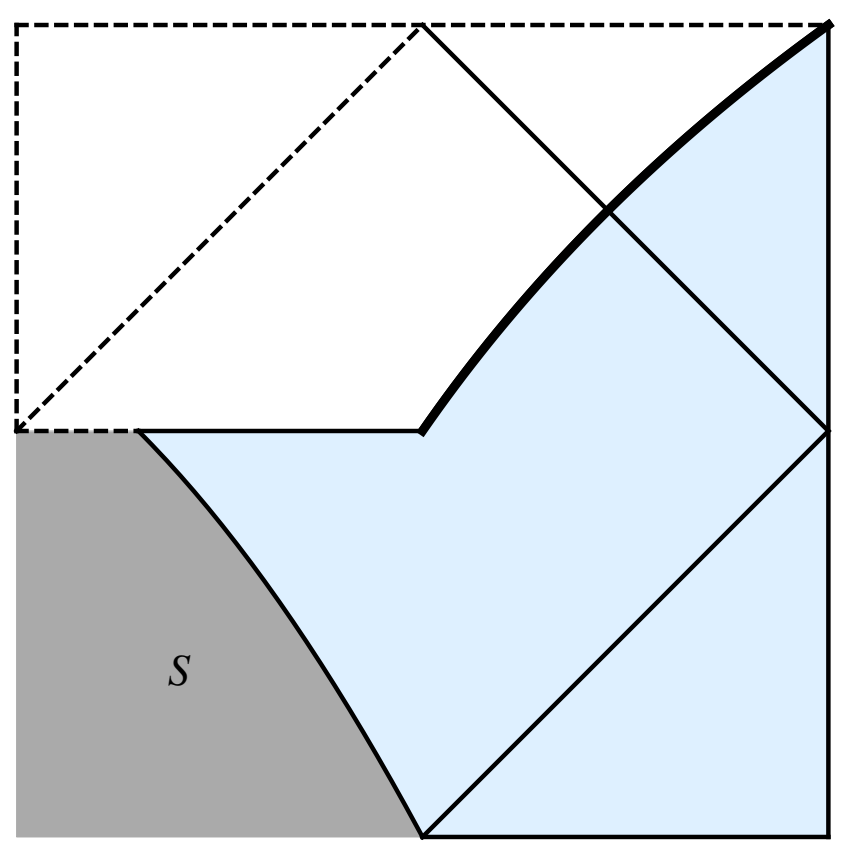

Figure 9. The Penrose diagram for a collapsing lump of matter $S$ in the false vacuum region. The space-time outside of the collapsing lump is made up of just a finite number of regions from the fully extended space-time.

The black hole emits Hawking radiation at a rate depending on fundamental particle masses and spins. The total decay rate for a subset of the standard model was evaluated by Page, [31]. If we set $\Gamma_{H}=\dot{M} / M$, then

$$
\Gamma_{H} \approx 3.6 \times 10^{-4}\left(G^{2} M_{C}^{3}\right)^{-1}
$$

The ratio of the two decay rates is

$$
\frac{\Gamma_{*}}{\Gamma_{H}} \approx 3.9 \times 10^{3}\left(G M_{C}^{2}\right)^{3 / 2} e^{-4 \pi G M_{C}^{2}} .
$$

Since this ratio is small for black holes larger than the Planck mass $G M_{C}^{2}>1$, these black holes decay before they can nucleate the false vacuum decay. The enhanced rate is relevant mostly for masses $M \ll M_{C}$ or for cases where the Hawking evaporation is suppressed, for example by de Sitter background radiation. Alternately, another physical mechanism, such as accretion of a slowly rolling scalar, [34], could negate the evaporative process (although this would simultaneously cause the tunnelling rate to drop).

Another interesting generalization of our study would be to explore the effect in extra dimensional scenarios, such as [35, 36], where the Planck mass can drop substantially, leading to interesting new black hole phenomenology, (for a review see [37]). The CDL instanton has been generalised to a braneworld construction, [38], however, to include black holes in this picture would lead to the usual impasse of a lack of an analytic exact 
solution for the C-metric (see [39] for a review of these issues, and [40] for recent numerical results).

Alternatively, there are many string theoretic models with 'large' or warped extra dimensions, such as KKLT, [41], or LVS (large volume scenarios, [42]), in which a CDL type of computation has been used to argue the long lifetime of a metastable dS vacuum (see also [43, 44]). For KKLT, the tension of the bubble wall lies outside the range allowed by the static patch construction: $2 \bar{\sigma} \ell \gg 1$, [41]. Thus we cannot directly apply our results, however, the intriguing possibility remains that the existence of a black hole could act as a nucleation site for decompactification. We leave the construction of supercritical black hole instantons for future investigation.

\section{Acknowledgments}

We would like to thank Patrick Dorey for discussions. RG and IM are supported in part by STFC (Consolidated Grant ST/J000426/1). RG is also supported by the Wolfson Foundation and Royal Society, and by Perimeter Institute for Theoretical Physics. Research at Perimeter Institute is supported by the Government of Canada through Industry Canada and by the Province of Ontario through the Ministry of Research and Innovation. BW was supported by a Royal Commission for the Exhibition of 1851 Research Fellowship.

\section{A Conical deficit regularisation}

In this appendix we review the computation of a conical deficit action. Our assumption is that the metric has a specific product structure in which the conical deficit is in a 2plane, parametrized by local cylindrical coordinates, $\{\rho, \chi\}$, and the transverse space $H$ is independent of these coordinates as $\rho \rightarrow 0$. Since we are interested in near horizon geometries we will specify the metric to be

$$
d s^{2}=d \rho^{2}+A^{2}(\rho) d \chi^{2}+C^{2}(\rho) d \Omega_{H}^{2},
$$

although the argument is independent of the precise structure the sections transverse to $\{\rho, \chi\}$, provided $C^{\prime}(0)=0$. We define the area $\mathcal{A}$ of the conical defect to be $C(0)^{n}$ times the area given by the metric $d \Omega_{H}^{2}$, where $n$ is the dimension of $H$.

The idea is now to smooth out the conical deficit by taking a regular function $A$ such that $A^{\prime}(0)=1, A^{\prime}(\epsilon)=(1-\delta)$, where $2 \pi \delta$ is the deficit angle. Because $C(\rho)$ remains smooth, we may write $C=C_{0}+\rho^{2} C_{2}$, and hence compute the Ricci scalar in the vicinity of $\rho=0$ as:

$$
\begin{aligned}
\mathcal{R} & =-\frac{2 A^{\prime \prime}}{A}-\frac{2 n C^{\prime \prime}}{C}-\frac{2 n A^{\prime} C^{\prime}}{A C}+\frac{n(n-1)\left(1-C^{2}\right)}{C^{2}} \\
& \sim-\frac{2 A^{\prime \prime}}{A}-\frac{4 n C_{2}}{C_{0}}+\frac{n(n-1)}{C_{0}^{2}}+\mathcal{O}(\rho)
\end{aligned}
$$

We see that this is the sum of a regular part (the terms involving $C_{0}, C_{2}$ and $\mathcal{O}(\rho)$ ) and the $A^{\prime \prime} / A$ term which becomes unbounded as $\epsilon \rightarrow 0$, as $A^{\prime \prime}=\mathcal{O}\left(\left(A^{\prime}(\epsilon)-A^{\prime}(0)\right) / \epsilon\right)$. In 
computing the integral of the Ricci scalar over a small region around $\rho=0$ therefore, it is only this unbounded term which will contribute:

$$
\int d^{n} x \sqrt{g} \mathcal{R} \sim \mathcal{A}\left[A^{\prime}(0)-A^{\prime}(\epsilon)\right]+\mathcal{O}(\epsilon)=4 \pi \delta \mathcal{A}+\mathcal{O}(\epsilon)
$$

in agreement with [30], for example.

To compute the Gibbons-Hawking boundary term, note that the relevant inward pointing normal is $n=-d \rho$, with extrinsic curvature $K=\nabla_{a} n^{a}=-A^{\prime} / A-2 n C^{\prime} / C$, hence

$$
\int_{\rho=\epsilon} d \chi d \Omega_{H} A C^{n} K \sim-2 \pi \mathcal{A} A^{\prime}(\epsilon)+\mathcal{O}(\epsilon)=-2 \pi \mathcal{A}(1-\delta)+\mathcal{O}(\epsilon) .
$$

Combining these terms together, we see that the contribution of the deficit angle, $\delta$, cancels, and taking $\epsilon \rightarrow 0$, we are left with the overall action:

$$
I_{\mathcal{B}}=-\int d^{4} x \sqrt{g} \frac{\mathcal{R}}{16 \pi G}+\int d^{3} x \sqrt{h} \frac{K}{8 \pi G}=-\frac{\mathcal{A}}{4 G}
$$

\section{B The limits on $\kappa$}

The static solution is obtained when $U=U^{\prime}=0$ for some $\tilde{R}_{*},(\tilde{R}=R / \gamma)$ which gives two polynomial constraints:

$$
\begin{aligned}
\tilde{R}_{*}^{6}-\left(\kappa_{2}+\frac{\kappa_{1}}{2}\right) \tilde{R}_{*}^{3}-2 \kappa_{2}^{2} & =0 \\
\tilde{R}_{*}^{3}-\frac{2}{3} \tilde{R}_{*}+\kappa_{2}+\frac{\kappa_{1}}{2} & =0
\end{aligned}
$$

Consistency of the solution $\tilde{R}_{*}^{3}$ to the 'quadratic', and $\tilde{R}_{*}$ to the cubic then requires

$$
\begin{aligned}
\kappa_{1}=\kappa_{1}^{*}=\frac{1}{81}[1 & -\left(-1-5\left(27 \kappa_{2}\right)^{2}+\frac{\left(27 \kappa_{2}\right)^{4}}{2}+\frac{27 \kappa_{2}}{2}\left(4+\left(27 \kappa_{2}\right)^{2}\right)^{3 / 2}\right)^{1 / 3} \\
& \left.+\left(1+5\left(27 \kappa_{2}\right)^{2}-\frac{\left(27 \kappa_{2}\right)^{4}}{2}+\frac{27 \kappa_{2}}{2}\left(4+\left(27 \kappa_{2}\right)^{2}\right)^{3 / 2}\right)^{1 / 3}\right]
\end{aligned}
$$

which gives us an upper bound on $\kappa_{1}$.

To get a lower bound on $\kappa_{1}$, we use

$$
f_{+} \dot{\tau}_{+}=\frac{\kappa_{2}}{\tilde{R}^{2}}+\tilde{R}(1-2 \bar{\sigma} \gamma) \geq 0
$$

(the constraint from positivity of $\dot{\tau}_{-}$being weaker). This constraint is saturated when $\tilde{R}_{+}^{3}=-\kappa_{2} /(1-2 \bar{\sigma} \gamma)$. For $\bar{\sigma} \ell>1 / 2$, we must have $\kappa_{2}>0$, and $\tilde{R}_{+}$must be greater than the maximum allowed value of $\tilde{R}$, i.e. $\mathrm{U}\left(\tilde{\mathrm{R}}_{+}\right), \mathrm{U}^{\prime}\left(\tilde{\mathrm{R}}_{+}\right)<0$. Conversely, for $\bar{\sigma} \ell<1 / 2$, the nontrivial minimal value for $\kappa_{1}$ occurs for $\kappa_{2}<0$, and $\tilde{R}_{+}$must be less than the minimum allowed value of $\tilde{R}$, i.e. $\mathrm{U}\left(\tilde{\mathrm{R}}_{+}\right)<0, U^{\prime}\left(\tilde{R}_{+}\right)>0$. In each case, the range closes off when $\tilde{R}_{+}=\tilde{R}_{*}$, leading to the $\kappa$-limits:

$$
\begin{aligned}
\frac{4}{27} \geq \kappa_{2} \geq \kappa_{2, \min }(\bar{\sigma}) & =\frac{(2 \bar{\sigma} \gamma-1) \ell^{3}}{3 \sqrt{3} \gamma^{3}} \\
\kappa_{1}^{*} \geq \kappa_{1} \geq \kappa_{1, \min }\left(\kappa_{2}, \bar{\sigma}\right) & =\operatorname{Max}\left\{\frac{4 \kappa_{2} \bar{\sigma}^{2} \gamma^{2}}{(1-2 \bar{\sigma} \gamma)}+\left|\frac{-\kappa_{2}}{(1-2 \bar{\sigma} \gamma)}\right|^{1 / 3}, 0\right\}
\end{aligned}
$$

From the range of $\kappa_{2}$, we conclude that $\bar{\sigma} \ell \leq \sqrt{3} / 2$. 
At the critical point $\kappa_{2, \text { min }}$, the seed mass $M_{+}=M_{N}$, and the remnant mass $M_{-}=$ $\left(3-4 \bar{\sigma}^{2} \ell^{2}\right) M_{N} / 2$, hence the static bounce action is

$$
B_{*}=\frac{\pi\left[R_{N}^{2}-\left(2 G M_{-}\right)^{2}\right]}{G}=\frac{8}{27} \frac{\pi \ell^{2}}{G} \bar{\sigma}^{2} \ell^{2}\left(3-2 \bar{\sigma}^{2} \ell^{2}\right)
$$

Thus, although the bounce action does tend to zero as $\bar{\sigma} \rightarrow 0$, it does so far more slowly than $B_{C D L}$, which is proportional to $\bar{\sigma}^{4}$.

Open Access. This article is distributed under the terms of the Creative Commons Attribution License (CC-BY 4.0), which permits any use, distribution and reproduction in any medium, provided the original author(s) and source are credited.

\section{References}

[1] S.R. Coleman, The Fate of the False Vacuum. 1. Semiclassical Theory, Phys. Rev. D 15 (1977) 2929 [Erratum ibid. D 16 (1977) 1248] [INSPIRE].

[2] C.G. Callan Jr. and S.R. Coleman, The Fate of the False Vacuum. 2. First Quantum Corrections, Phys. Rev. D 16 (1977) 1762 [InSPIRE].

[3] A.H. Guth, The Inflationary Universe: A Possible Solution to the Horizon and Flatness Problems, Phys. Rev. D 23 (1981) 347 [InSPIRE].

[4] M.S. Turner and F. Wilczek, Might our vacuum be metastable?, Nature 298 (1982) 633 [INSPIRE].

[5] W.A. Hiscock, Can black holes nucleate vacuum phase transitions?, Phys. Rev. D 35 (1987) 1161 [INSPIRE].

[6] V.A. Berezin, V.A. Kuzmin and I.I. Tkachev, O(3) Invariant Tunneling in General Relativity, Phys. Lett. B 207 (1988) 397 [InSPIRE].

[7] V.A. Berezin, V.A. Kuzmin and I.I. Tkachev, Black holes initiate false vacuum decay, Phys. Rev. D 43 (1991) 3112 [INSPIRE].

[8] I.G. Moss, Black hole bubbles, Phys. Rev. D 32 (1985) 1333 [InSPIRE].

[9] C. Cheung and S. Leichenauer, Limits on New Physics from Black Holes, arXiv: 1309.0530 [INSPIRE].

[10] S.R. Coleman and F. De Luccia, Gravitational Effects on and of Vacuum Decay, Phys. Rev. D 21 (1980) 3305 [INSPIRE].

[11] H. Nariai, On some static solutions of Einstein's gravitational field equations in a spherically symmetric case, Sci. Rept. Tohoku Univ. 34 (1950) 160.

[12] H. Nariai, On a new cosmological solution of Einstein's field equations of gravitation, Sci. Rept. Tohoku Univ. 35 (1951) 46.

[13] S.W. Hawking and N. Turok, Open inflation without false vacua, Phys. Lett. B 425 (1998) 25 [hep-th/9802030] [INSPIRE].

[14] N. Turok and S.W. Hawking, Open inflation, the four form and the cosmological constant, Phys. Lett. B 432 (1998) 271 [hep-th/9803156] [INSPIRE].

[15] G.W. Gibbons and S.W. Hawking, Classification of Gravitational Instanton Symmetries, Commun. Math. Phys. 66 (1979) 291 [INSPIRE]. 
[16] J. Garriga and A. Megevand, Decay of de Sitter vacua by thermal activation, Int. J. Theor. Phys. 43 (2004) 883 [hep-th/0404097] [INSPIRE].

[17] E. Farhi, A.H. Guth and J. Guven, Is It Possible to Create a Universe in the Laboratory by Quantum Tunneling?, Nucl. Phys. B 339 (1990) 417 [inSPIRE].

[18] W. Fischler, D. Morgan and J. Polchinski, Quantum Nucleation of False Vacuum Bubbles, Phys. Rev. D 41 (1990) 2638 [inSPIRE].

[19] W. Fischler, D. Morgan and J. Polchinski, Quantization of False Vacuum Bubbles: A Hamiltonian Treatment of Gravitational Tunneling, Phys. Rev. D 42 (1990) 4042 [InSPIRE].

[20] A. Aguirre and M.C. Johnson, Dynamics and instability of false vacuum bubbles, Phys. Rev. D 72 (2005) 103525 [gr-qc/0508093] [INSPIRE].

[21] A. Aguirre and M.C. Johnson, Two tunnels to inflation, Phys. Rev. D 73 (2006) 123529 [gr-qc/0512034] [INSPIRE].

[22] W. Israel, Singular hypersurfaces and thin shells in general relativity, Nuovo Cimento Soc. Ital. Phys. B 44 (1966) 4349.

[23] P. Bowcock, C. Charmousis and R. Gregory, General brane cosmologies and their global space-time structure, Class. Quant. Grav. 17 (2000) 4745 [hep-th/0007177] [INSPIRE].

[24] F. Mellor and I. Moss, Black Holes and Quantum Wormholes, Phys. Lett. B 222 (1989) 361 [INSPIRE].

[25] F. Mellor and I. Moss, Black Holes and Gravitational Instantons, Class. Quant. Grav. 6 (1989) 1379 [INSPIRE].

[26] F. Dowker, J.P. Gauntlett, D.A. Kastor and J.H. Traschen, Pair creation of dilaton black holes, Phys. Rev. D 49 (1994) 2909 [hep-th/9309075] [InSPIRE].

[27] A.R. Brown and E.J. Weinberg, Thermal derivation of the Coleman-De Luccia tunneling prescription, Phys. Rev. D 76 (2007) 064003 [arXiv:0706.1573] [inSPIRE].

[28] R.P. Geroch and J.H. Traschen, Strings and Other Distributional Sources in General Relativity, Phys. Rev. D 36 (1987) 1017 [INSPIRE].

[29] S.W. Hawking and G.T. Horowitz, The Gravitational Hamiltonian, action, entropy and surface terms, Class. Quant. Grav. 13 (1996) 1487 [gr-qc/9501014] [INSPIRE].

[30] D.V. Fursaev and S.N. Solodukhin, On the description of the Riemannian geometry in the presence of conical defects, Phys. Rev. D 52 (1995) 2133 [hep-th/9501127] [INSPIRE].

[31] D.N. Page, Particle Emission Rates from a Black Hole: Massless Particles from an Uncharged, Nonrotating Hole, Phys. Rev. D 13 (1976) 198 [InSPIRE].

[32] C. Charmousis and R. Gregory, Axisymmetric metrics in arbitrary dimensions, Class. Quant. Grav. 21 (2004) 527 [gr-qc/0306069] [InSPIRE].

[33] C. Charmousis, Dilaton space-times with a Liouville potential, Class. Quant. Grav. 19 (2002) 83 [hep-th/0107126] [INSPIRE].

[34] S. Chadburn and R. Gregory, Time dependent black holes and scalar hair, arXiv:1304.6287 [INSPIRE].

[35] N. Arkani-Hamed, S. Dimopoulos and G.R. Dvali, The Hierarchy problem and new dimensions at a millimeter, Phys. Lett. B 429 (1998) 263 [hep-ph/9803315] [INSPIRE]. 
[36] L. Randall and R. Sundrum, A Large mass hierarchy from a small extra dimension, Phys. Rev. Lett. 83 (1999) 3370 [hep-ph/9905221] [INSPIRE].

[37] P. Kanti, Black holes in theories with large extra dimensions: A Review, Int. J. Mod. Phys. A 19 (2004) 4899 [hep-ph/0402168] [INSPIRE].

[38] R. Gregory and A. Padilla, Brane world instantons, Class. Quant. Grav. 19 (2002) 279 [hep-th/0107108] [INSPIRE].

[39] R. Gregory, Braneworld black holes, Lect. Notes Phys. 769 (2009) 259 [arXiv:0804.2595] [INSPIRE].

[40] P. Figueras and T. Wiseman, Gravity and large black holes in Randall-Sundrum II braneworlds, Phys. Rev. Lett. 107 (2011) 081101 [arXiv:1105.2558] [INSPIRE].

[41] S. Kachru, R. Kallosh, A.D. Linde and S.P. Trivedi, de Sitter vacua in string theory, Phys. Rev. D 68 (2003) 046005 [hep-th/0301240] [INSPIRE].

[42] V. Balasubramanian, P. Berglund, J.P. Conlon and F. Quevedo, Systematics of moduli stabilisation in Calabi-Yau flux compactifications, JHEP 03 (2005) 007 [hep-th/0502058] [INSPIRE].

[43] A. Westphal, Lifetime of Stringy de Sitter Vacua, JHEP 01 (2008) 012 [arXiv:0705.1557] [INSPIRE].

[44] S. de Alwis, R. Gupta, E. Hatefi and F. Quevedo, Stability, Tunneling and Flux Changing de Sitter Transitions in the Large Volume String Scenario, JHEP 11 (2013) 179 [arXiv: 1308.1222] [INSPIRE]. 